\title{
REXUS-25 Rocket Flight of a CubeSat Cosmic-Ray Detector
}

\author{
B. Pont ${ }^{* a}$, J. Beurskens ${ }^{a}$, J. Dalderup ${ }^{b}$, P. Dolron ${ }^{a}$, J. Gubbels ${ }^{b}$, J.R. Hörandel ${ }^{a, c, d}$, \\ R. Jordans ${ }^{a, b}$, H. Pourshaghaghi ${ }^{a}, b$, D. Szálas-Motesiczky ${ }^{a}$, T. van Vliet $^{b}$, \\ M. Wijtvliet ${ }^{b}$, J. Witteman ${ }^{b}$ \\ ${ }^{a}$ Radboud University, P.O. Box 9010, NL-6500 GL Nijmegen, The Netherlands \\ ${ }^{b}$ Eindhoven University of Technology, P.O. Box 513, NL-5600 MB Eidhoven, The Netherlands \\ ${ }^{c}$ NIKHEF, Science Park Amsterdam, 1098 XG Amsterdam, The Netherlands \\ ${ }^{d}$ Vrije Universiteit Brussel, Pleinlaan 2, 1050 Brussels, Belgium \\ E-mail: b.ponteastro.ru.nI
}

In recent years CubeSats have been revolutionizing research in space, enabling low-budget, small-scale, and fast-development of projects. The Rocket Experiments for University Students (REXUS) program provides students from higher education opportunities to perform their scientific and technological experiments on a sounding rocket launching to space.

A compact cosmic-ray detector, build to CubeSat specifications, has been designed to detect light produced in a scintillating material when cosmic rays pass through. At only $1 \mathrm{dm}^{3}$ in size and operating on only 1 Watt, this REXUS payload as part of the Payload for Radiation measurement and Radio-interferometry in Rockets (PR3), has measured the charged-particle rate going up in the atmosphere and into space.

Besides providing a chance for students to work on reproducing the historical discoveries of Hess, Pfotzer, Regener, Van Allen, and others in the last century, it has also been an outreach opportunity to engage the general public with astroparticle physics. But above all, it is a proof of concept for compact cosmic-ray detectors in orbit. Where the worldwide network of neutron detectors is able to monitor the cosmic-ray flux at ground level, and detectors on the ground and in space detect cosmic rays locally, global near-real-time coverage of cosmic-ray flux above the atmosphere is only feasible with an ensemble of small and cheap detectors like the one presented here.

Details on the detector design and performance, and the results from the rocket flight will be presented.

36th International Cosmic Ray Conference -ICRC2019-

July 24th - August 1st, 2019

Madison, WI, U.S.A.

${ }^{*}$ Speaker. 


\section{Introduction}

On March 11, 2019 the REXUS-25 rocket launched from the Esrange Space Center near Kiruna, Sweden in the high Arctic. On board was the student project PR3 (Payload for Radiation measurement and Radio-interferometry in Rockets) from two Dutch universities Radboud University Nijmegen and Eindhoven University of Technology. The flight was nominal, reached an apogee of $82 \mathrm{~km}$, and the payload landed again safely 15 minutes later.

On board was a prototype compact satellite cosmic-ray detector. The goal was to test the effectiveness of the consumer-grade hardware and its robustness to the extreme conditions of a rocket launch and vacuum conditions of space. This launch was a part of the ongoing efforts to develop a CubeSat cosmic-ray detector for orbital flight.

During it's ascent it measured the secondary cosmic rays from the extensive air showers of high-energy cosmic-rays and after leaving a big part of the atmosphere the primaries.

In the following sections the hardware, its performance during the flight will be described, and the results from the measurements will be presented.

\section{A CubeSat Cosmic-Ray Detector}

In 1999 the California Polytechnic State University and Stanford University developed a standard for a small cubic satellite, CubeSat for short, that can be launched in bulk as secondary payload with a larger project. The small scale of the satellite would enable universities and even student projects access to space, mostly in low Earth orbit, for a relatively low cost. Small-scale satellites with a standardized format also allow for fast development of projects with the additional advantage of a fast innovation cycle.

The small design however also brings limitations. It has to mostly rely on solar panels for power which at only $1 \mathrm{dm}^{2}$ facing the Sun at any time provides only little power. A CubeSat typically has to run on as little as $6 \mathrm{~W}$ of which about $1 \mathrm{~W}$ is left for the experiment itself [1]. For this reason our detector has been designed to operate on $0.5-1 \mathrm{~W}$.

The bulk of the detector consists of a plastic scintillator block (NE110) with dimensions $74 \times 74 \times 50 \mathrm{~mm}^{3}$. Two optical fibers run through the center to two Avalanche Photo Diodes (APD) mounted at the bottom. Figure 1a shows the detector in its launch configuration. Figure $1 \mathrm{~b}$ is a simplified schematic drawing of the detector. The main advantage of APDs over standard photomultiplier tubes is that by using modern solid state techniques it can be made smaller, requirs less power (lower voltages), has faster responses (process a detection faster), and it will be less delicate so that it can handle mechanical shocks that would break PMTs. The maximum sensitivity of the APDs coincides with the peak of the scintillator emission spectrum $(\lambda=435 \mathrm{~nm})$, optimizing the detection efficiency. The APDs are mounted separately to minimize the risk of mechanical shocks affecting the connection between APDs and optical fibers. From the APDs connector wires run to the main electronics board. There the signal of the sensors is amplified and compared to a threshold set as to reject the majority of the (thermal) noise. If both sensors have a signal above threshold coinciding within a $10 \mathrm{~ns}$ time window an event is logged.

The amount of thermal noise increases with both, temperature and APD voltage. The detection efficiency of real signals increases with temperature and voltage. It is thus a balancing game 


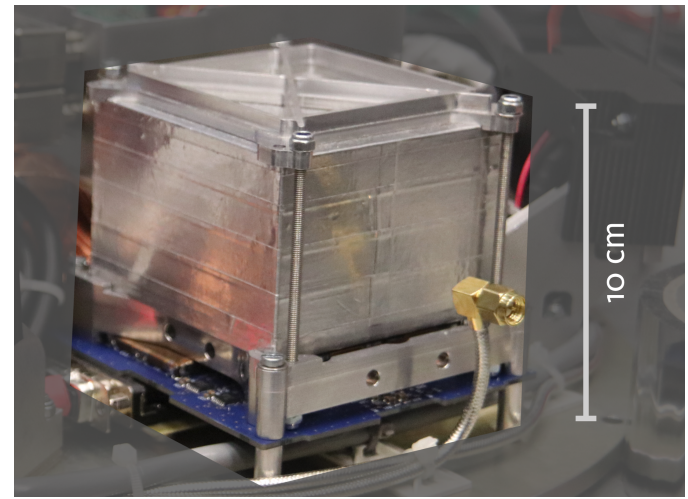

(a)

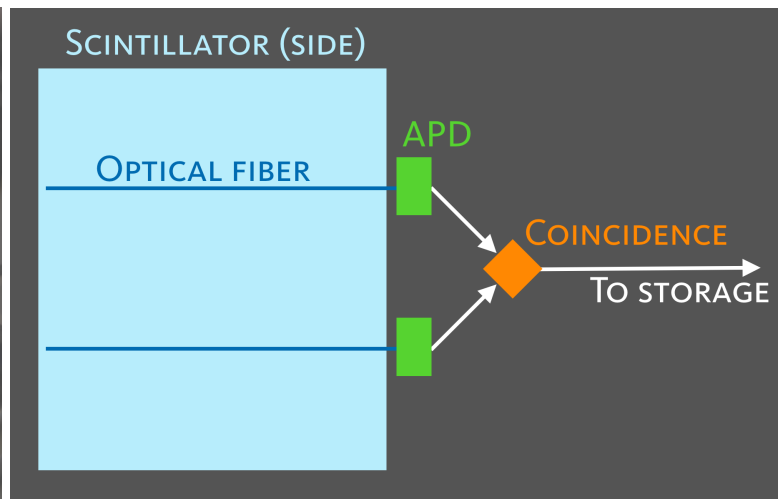

(b)

Figure 1: (a) Picture of the cosmic-day detector during pre-flight checks. Mounted above the electronics board (blue) are the light-shielded APD sensors and the light-shielded scintillator block. (b) Schematic drawing of the detector. Light produced in the scintillator is channeled through optical fibers towards two APD sensors that record a count if they are in coincidence.

between noise reduction and increasing efficiency. Prior to flight rudimentary measurements with a reference particle detector were performed to optimize this for operation above the noise floor in a range of temperatures between 20 to 50 degrees Celsius. These resulted in detection efficiency of around $40 \%$ at $25^{\circ} \mathrm{C}$ and around $10 \%$ at $50^{\circ} \mathrm{C}$. Due to time constrains of the rocket launch window a detailed efficiency characterization, as was done for an earlier prototype[2], had to be executed in this simplified form. Measuring the cosmic-ray rate is sufficient for the goals of this flight.

\section{The REXUS Program and the PR3 Project}

\subsection{The REXUS Program}

The REXUS project (Rocket EXperiments for University Students) is a project run by the European Space Agency (ESA), the Swedish National Space Board (SNSB), and the German Aerospace Centre (DLR). The aim of the project is to make space research available for university students. Each year two sounding rockets are launched from the Esrange Space Centre in northern Sweden, up to altitudes between 70 and $100 \mathrm{~km}$. The sounding rockets carry experiments designed, build, and tested by the students.[3]

\subsection{The PR3 Project}

A joint team of the Eindhoven University of Technology and Radboud University students, developed the PR3 payload (Payload for Radiation measurement and Radio-interferometry in Rockets [4]) for a REXUS rocket that was launched in March 2019 as part of the REXUS-25 campaign. This payload consisted of two experiments. One experiment aimed to track the position of the rocket to sub-cm accuracy using radio-interferometry. The other experiment aimed at measuring cosmic radiation and consisted of two complementary sub-experiments. CCD and CMOS camera sensors measured low-energy cosmic rays to study their potential as ultra-compact radiation monitoring equipment for future space-based missions. Secondly, and the focus of this proceeding, 
the scintillator cosmic-ray detector build to CubeSat specifications as a proof-of-concept to evaluate the performance of the hardware for a future CubeSat flight into orbit. It also functioned as a reference detector for the camera sensors.

\subsection{Flight Conditions}

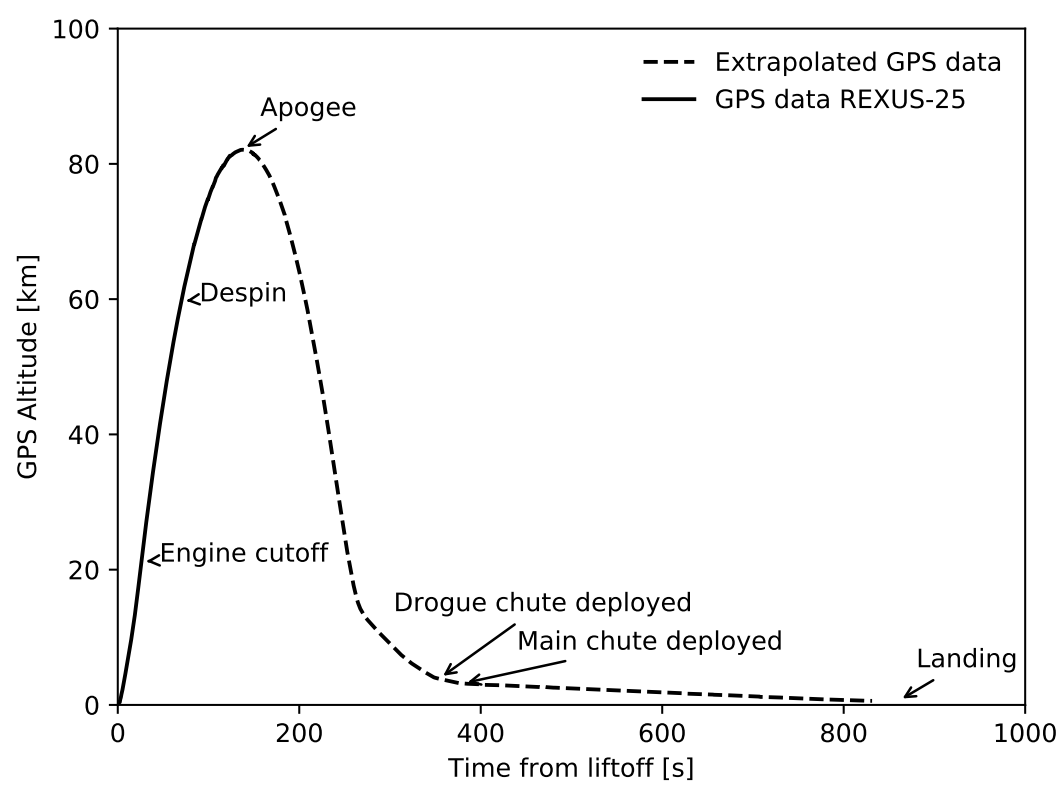

Figure 2: GPS altitude of the rocket versus time from liftoff. The main events during flight are highlighted. Because of GPS loss of signal the path after apogee is modeled based on previous flights with similar payload characteristics.

At the moment of launch the weather conditions at ground were favorable showing a temperature of $-9^{\circ} \mathrm{C}$, clear skies, and minimal wind. Inside the rocket the temperature was stable at around $30^{\circ} \mathrm{C}$. Due to the short flight duration and heating from the electronics this temperature remained essentially stable throughout the flight.

Figure 2 shows the altitude of the rocket as a function of time after launch. The total flight time was around 15 minutes of which 5 minutes were above $10 \mathrm{~km}$ and about 2 minutes above $60 \mathrm{~km}$. An apogee of just above $82 \mathrm{~km}$ was reached.

At a height of $21 \mathrm{~km}$, at $\mathrm{T}+26 \mathrm{~s}$ the engine was cut-off. After atmospheric drag became insignificant a de-spinning maneuver was performed at around $\mathrm{T}+70 \mathrm{~s}$. After that the rocket is essentially in a parabolic free-fall rotating freely for about 2-3 minutes until atmospheric drag builds up again. A drogue chute is deployed to stabilize the rocket followed by the main parachute deployment at around $3 \mathrm{~km}$ from the ground.

The rocket was launched from a latitude of $67^{\circ} \mathrm{N}$ where the magnetic field is almost pointing downward at a declination of roughly $10^{\circ}$ East and an inclination of around $77^{\circ}$ downwards.[5] Because of this also many low-energy cosmic-ray particles are to be expected that are funneled down along the magnetic field lines. 


\section{Results}

\subsection{Detector Performance}

The detector performed nominally during the entirety of the flight. Prior to the flight the detector passed vibration and vacuum tests. During the flight shock accelerations of up to $60 \mathrm{G}$ were recorded which did not affect operation or measurements of the detector itself.

The main means of data recording was done via the telemetry stream sent down from the rocket. It recorded the detected cosmic-ray rate during the flight in $0.8 \mathrm{~s}$ intervals. Build-in redundancy prevented loss of data packages. Secondly, data consisting of each detected scintillator coincidence signal with a nanosecond timestamp, detector voltage settings, and temperature on the electronics board was also logged to an on-board memory card. However, technical difficulties with the stability of a connector elsewhere outside our detector resulted in much of this additional data being corrupted. The following sections thus describe the data recorded via the telemetry stream.

\subsection{Cosmic-Ray Rate and Atmospheric Depth}

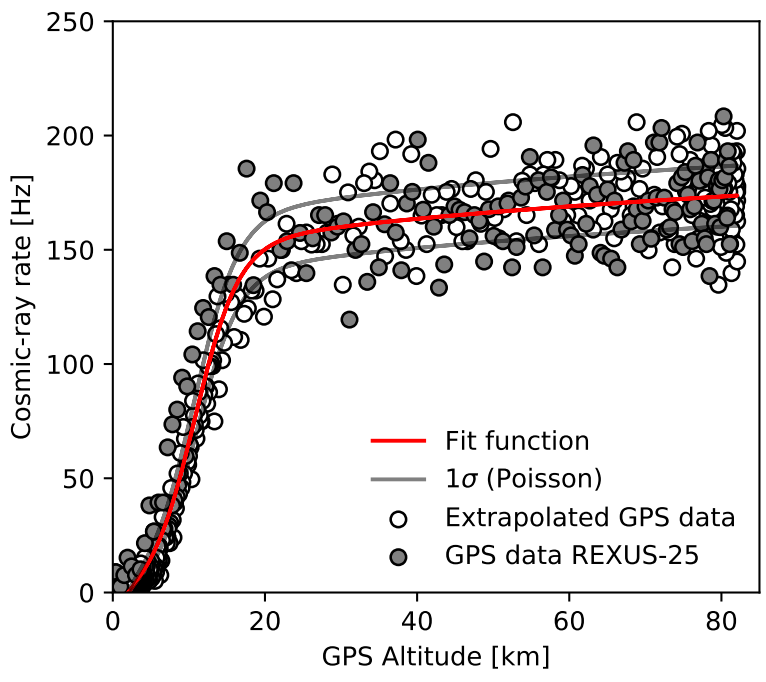

(a)

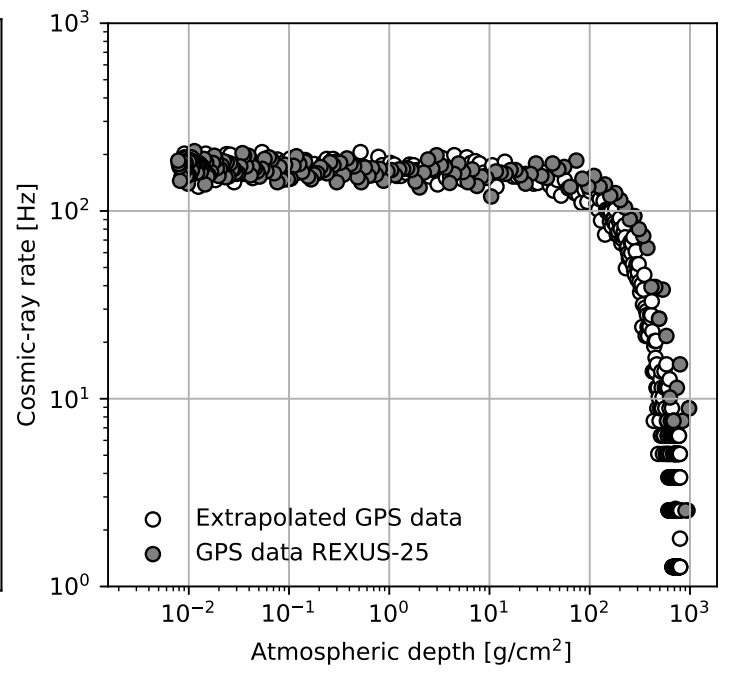

(b)

Figure 3: (a) Measured cosmic-ray rate as a function of GPS altitude (grey) and as a function of the aforementioned GPS extrapolation after apogee (white). The grey lines illustrate a $1 \sigma$ Poisson error around the red line. (b) Cosmic-ray rate as function of atmospheric depth. The atmospheric depth is taken from GDAS at the time and location of the launch. The GDAS data is coupled via the GPS altitude to the cosmic-ray rate. It thus also relies on the GPS altitude extrapolation.

The number of secondary particles produced in extensive air showers close to the equator typically reach a maximum around $15 \mathrm{~km}$; the Pfotzer maximum.[6] At lower altitudes the flux decreases as absorption and decay thins the showers. At altitudes above the maximum a stable plateau will be reached where the secondary particles have not yet formed and only the primaries are detected. The exact position of the Pfotzer maximum depends on the latitude. Near the equator this lies around $15 \mathrm{~km}$ while near the North Pole this lies closer to $20 \mathrm{~km}$. The latitude has another effect; the magnetic field orientation determines the rigidity cutoff of cosmic rays. Near the poles 


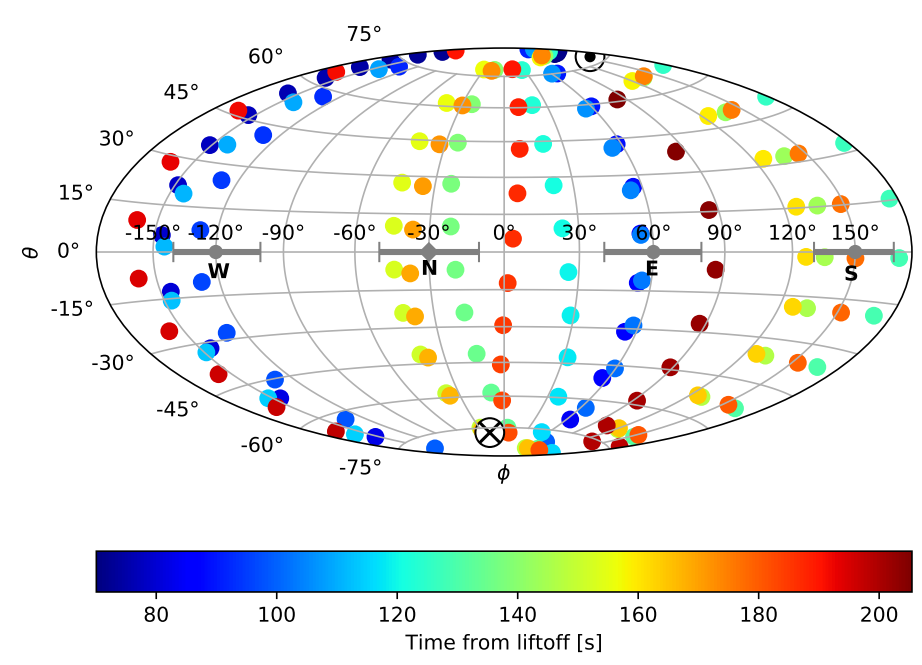

Figure 4: Direction of the rocket on the sky for each time step above $60 \mathrm{~km}$. The colorbar shows the time from liftoff. The upper hemisphere is the sky above the local horizon. The cardinal directions determined from camera footage are shown with their uncertainty. The two circles near the poles represent the direction of the magnetic field.

lower energy primary particles are not deflected away and thus can dominate over the secondary particles of the Pfotzer maximum during a solar mimimum when the primary cosmic-ray flux is even higher. See for example [7] for CRAC:CRII modeling of these effects.

Because our measurement were done at a latitude of $67^{\circ}$ during a solar minimum the expected particle flux profile should rise to a plateau without a clearly visibly Pfotzer maximum peak. Figure $3 \mathrm{a}$ shows the measured cosmic-ray rate as function of altitude. It is consistent with these expectation. Also consistent is the altitude of about $20 \mathrm{~km}$ where the maximum rate is reached. Note that part of the GPS altitude had to be extrapolated based on flight trajectories of previous flights with similar payload characteristics because of a loss of GPS signal by the rocket service module. The small offset in measured rate at lower altitudes between the ascent and descent phases is most likely caused by imperfections in the extrapolation.

The cosmic-ray flux is primarily a function of the amount of atmosphere encountered by the particles and not altitude. We use the Global Data Assimilation System (GDAS) to get the atmospheric depth profile for the time and location of the flight. Prior to flight only a rudimentary measurement of efficiency could be made (see section 2). For this reason a rate is shown instead of a flux. Fully characterizing the efficiency and detection threshold for different energies will be work for the future.

\subsection{Arrival Directions above the Atmosphere}

To determine the nature of the measured cosmic rays the arrival direction of the particles is reconstructed. Low-energy particles are expected to follow primarily the direction of the magnetic field while higher energy particles would arrive more uniformly.

At this point we want to stress that this detector was not designed with direction reconstruction in mind. It is still able to do this because the scintillator block in the detector is not symmetric but thinner by almost a factor of 2 in one of its three axes. The measured rate versus time seems to show 

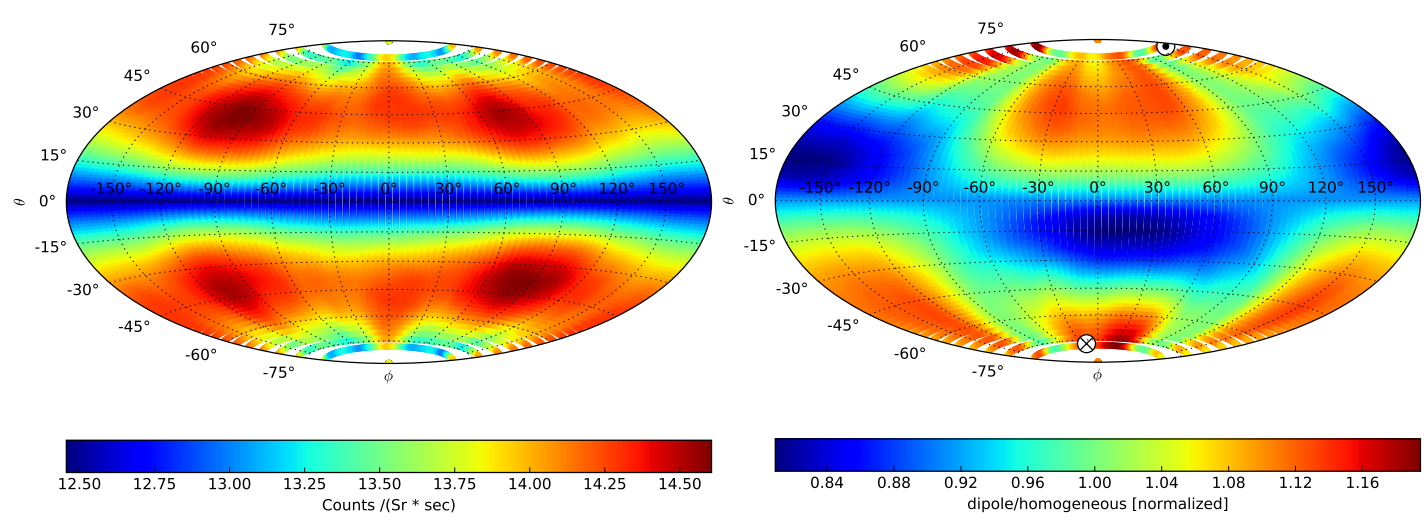

(a)

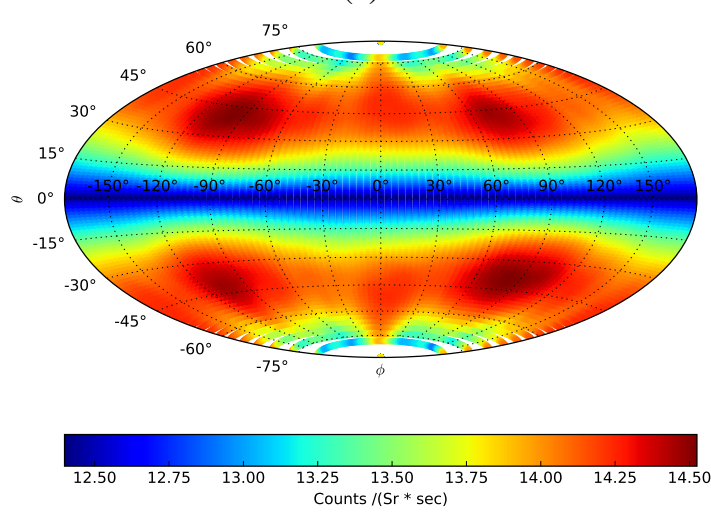

(c)

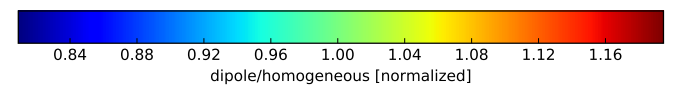

(b)

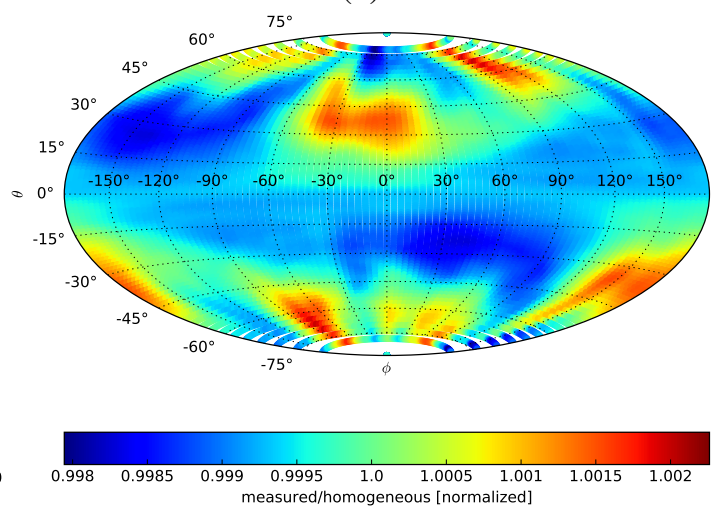

(d)

Figure 5: Skymaps of the cosmic-ray intensity. (a) Homogeneous sky model with noise as seen by the detector. (b) Normalized dipole sky model (average of 20 noise iterations).(c) Measured sky map as seen by the detector. (d) Reconstructed normalized dipole component from data (average of 20 noise iterations)

hints of a fluctuating pattern at high altitudes larger than expected from Poissonian fluctuations. For the period of time that the rocket is in parabolic free-fall it rotates around all three principal axes scanning across the sky (figure 4). There is thus directional information to be extracted if the arrival directions are indeed non-uniform. The orientation of the rocket is reconstructed using the gyroscopic data of the yaw, pitch, and roll rates integrated over time and transformed from the rocket frame to a local sky frame fixed to the ground. The validity of this method was verified by comparing the reconstructed orientation to on-board video footage of the flight. Using the orientation of the detector the effective surface area is known in each direction on the sky at any point in time. This effective surface area is used as arrival direction probability to smear out the measured cosmic-ray rate over the sky for each time step above $60 \mathrm{~km}$.

Figure $5 \mathrm{c}$ shows the number of counts per steradian per second on each point of the sky as reconstructed from the measurements. The effective area of the scintillator is the same on opposite sides of the sky because of the detector geometry. For this reason it can't distinguish top from bottom, right from left, and front from back resulting in mirror images of parts of the sky. Disentangling a uniform background from a preferred direction is limited here to looking for a simple 
dipole component (looks the same as a single hotspot because of the asymmetric detector geometry) modelling particles arriving aligned with the magnetic field. We model what a pure uniform and pure dipole sky with the same integrated signal as our measurements would look like as seen by our detector. For these models first a theoretical sky map is generated. Next the path on the sky from the flight (figure 4) is used to extract the rate that would be measured for the model sky at those points in the flight. The skymap is then reconstructed in the same way as was done for the measurements. Figure 5a shows a uniform background with Poisson fluctuations. Figure 5b shows the reconstructed dipole sky, but because of the reconstruction method part is smeared over the sky, the skymap is normalized with the average of 20 uniform sky models with noise so that the deviation from uniform above the noise becomes visible.

To see if such a dipole is present above the noise level we again remove 20 times a uniform model and look at the average of the residuals (figure 5d). Comparing this to the model dipole aligned with the magnetic field (figure $5 \mathrm{~b}$ ) shows the dipole component reconstructed from measurements is well-aligned with the modelled dipole within our uncertainty of the magnetic field direction. Our dipole component (measured) has deviations from the background of $1 \%$ compared to a pure dipole model. The reason for such a small fraction might be the partly the simple model for the shape of the dipole representing the magnetic field or the fact that our assumption of a homogeneous background is not sufficient to describe the sky. Also the arrival direction probability distribution assigned to each time step does not account for detection thresholds and would required further study. This is beyond the scope of this work that used this skymap reconstruction to verify the operation of the detector.

\section{Conclusion}

We report the development of a compact cosmic-ray detector and the operation tested during an sub-orbital sounding rocket flight. The detector itself performed as expected for the full duration of the flight and was able to measure the cosmic-ray rate up to $82 \mathrm{~km}$ altitude. The altitude-rate profile is compatible with expectations from simulations. Using the asymmetrical detector shape a skymap could be reconstructed consistent with a uniform background with a dipole component aligned close to the direction of the magnetic field supporting the idea of the presence of lower energy cosmic rays.

\section{References}

[1] K. Woellerta, Advances in Space Research 47(4):663-684, feb 2011.

[2] B. Pont, Development of a CubeSat Cosmic-Ray Detector, Master Thesis, Raboud University Nijmegen, oct 2016.

[3] REXUS User Manual http://rexusbexus.net/rexus/rexus-user-manual/

[4] PR3 project, https://www.ru.nl/astrophysics/radboud-radio-lab/projects/pr3/

[5] E. Thébault et. al. Earth, Planets and Space 201567:79, https://doi.org/10.1186/s40623-015-0228-9

[6] G. Pfotzer, Dreifachkoinzidenzen der Ultrastrahlung aus vertikaler Richtung in der Stratosphere, Z. Phys. 102, 23-58, 1936.

[7] M. Calisto, I. Usoskin, E. Rozanov, T. Peter, Atmos. Chem. Phys., 11, 4547-4556, 2011. 